\title{
Studies of Response of Metal - Porous Silicon Structures to Microwave Radiation
}

\author{
J. Stupakova ${ }^{a}$, S. Ašmontas ${ }^{b}$, J. Gradauskas $^{b, a}$, \\ V. ZagadskiJ ${ }^{a}$, E. Shatkovskis ${ }^{a, b, *}$ AND A. SuŽIedĖlis ${ }^{b, a}$ \\ ${ }^{a}$ Vilnius Gediminas Technical University, Saulettekio 11, 10223, Vilnius, Lithuania \\ ${ }^{b}$ Semiconductor Physics Institute, Gostauto 11, 01108, Vilnius, Lithuania
}

(Received July 10, 2006)

\begin{abstract}
Structures containing layers of porous silicon with two metal contacts are investigated. Porous silicon is manufactured by anodizing $p$-type crystalline silicon plates of resistivity of $0.4 \Omega \mathrm{cm}$. Contacts for the samples are made by additional boron doping of the surface and by thermal evaporation of aluminium. Resistance and current-voltage characteristics are investigated. Response of the porous silicon layer containing structures under action of pulsed microwave radiation was investigated for the first time. The origin of the response is discussed.
\end{abstract}

PACS numbers: 72.30.+q, 78.55.Mb, 85.30.De

\section{Introduction}

Canham's paper on porous silicon (PSi) has caused an increased interest to its unique properties [1]. In one of the first papers devoted to electroluminescence studies in metal - porous silicon (M/PSi) structure, it was underlined that on the interface of PSi with $n$-type indium - thin oxide there existed an area of spatial charge and a potential barrier [2]. Rectifying current-voltage $(I-V)$ characteristic shape was typical of such diode structures. Similar $I-V$ characteristics of the M/PSi structures were established in other papers, too [3, 4]. It was noticed that potential barrier height depended on the kind of metal having a contact with $\mathrm{PSi}$, e.g. for gold it was $(0.7-0.8) \mathrm{eV}$, and for nickel $-0.9 \mathrm{eV}$ [3]. Some papers, however, showed alternative results and models of current flow in M/PSi structures. Authors judge on more complex structure of potential barriers. A model of double injection both through Schottky barrier and through interface

*corresponding author; e-mail: eusat@pfi.lt 
$\mathrm{PSi} / \mathrm{c}-\mathrm{Si}$ was suggested to explain power dependence of current at high voltages $[5,6]$. Aluminium is usually used to create ohmic contacts to c-Si. From temperature dependence studies of $I-V$ characteristics and impedance of aluminium/ $p$-type PSi structures [7], the authors showed not only the presence of potential barrier in the interface PSi/c-PSi, but also found an inverse layer with $n$-type conductivity. Thus M/PSi structures represent rather a complex formation with various characteristics depending on properties of initial material and on parameters and sequence of technological process. One of specific PSi properties, controlled porosity, is used to reduce dielectric losses in the microwave (MW) devices [8]. Papers on application of PSi to register or sense microwave radiations are not found. We have investigated $\mathrm{Al} / \mathrm{PSi}$ structures with intent to sense microwave radiation, and to get more information about potential barriers in M/PSi layers. Basic peculiarities of this action are established for the first time.

\section{Experimental}

Plates of $p$-type, boron doped, crystalline silicon (c-Si) of resistivity of $0.4 \Omega \mathrm{cm}$ and orientation (100) were used for PSi formation, by electrochemical etching in a mix of fluoric acid with ethanol in the ratio of 1:2 used as electrolyte. A full cycle of etching consisted of two phases: the density of anodic current was $10 \mathrm{~mA} / \mathrm{cm}^{2}$ and $80 \mathrm{~mA} / \mathrm{cm}^{2}$, and etching time was 5 and 10 minutes, respectively for each phase. More dense auxiliary $\mathrm{PSi}^{*}$ layer was formed in the first phase. From the density of anodic current [9], the porosity of the auxiliary layer was estimated to be about $55 \%$, and its thickness was about $3-5 \mu \mathrm{m}$. The basic layer of porous silicon $\left(\mathrm{PSi}^{* *}\right)$ was formed in the second phase. Its thickness was estimated to be about $20 \mu \mathrm{m}$, and porosity of it was about $75 \%$. For ohmic contacts, the surface of the plates was additionally boron-doped by boron diffusion, followed by thermal evaporation of aluminium in vacuum and subsequent annealing at a temperature of $450^{\circ} \mathrm{C}$ in nitrogen atmosphere. In contrast to the background contacts, areas of aluminium of $100 \mu \mathrm{m}$ in diameter were set our PSi instead of a continuous layer. The samples were cut out of the plates in the size of $0.5 \times 0.6 \mathrm{~mm}^{2}$. Measurements of resistance and $I-V$ characteristics were carried out by a universal measurer "Agilent 4156C". Microwave measurements were carried out in rectangular waveguide at $10 \mathrm{GHz}$ frequency, with radiation modulated in $2 \mu$ s pulses at a repetition rate of $40 \mathrm{~Hz}$. The signal was measured from the background contact whereas the $\mathrm{Al}$ contact platform to $p^{+}-\mathrm{PSi}^{*}$ layer was earthed. The response signals were tested by an oscillograph "Agilent 5464-2A".

\section{Results and discussion}

Scanning electronic microscope (SEM) microphotograph of PSi layer is shown in Fig. 1. The thickness of an auxiliary $\mathrm{PSi}^{*}$ layer is close to above evaluation. The basic $\mathrm{PSi}^{* *}$ layer is composed of nanometric scale silicon strings. The 


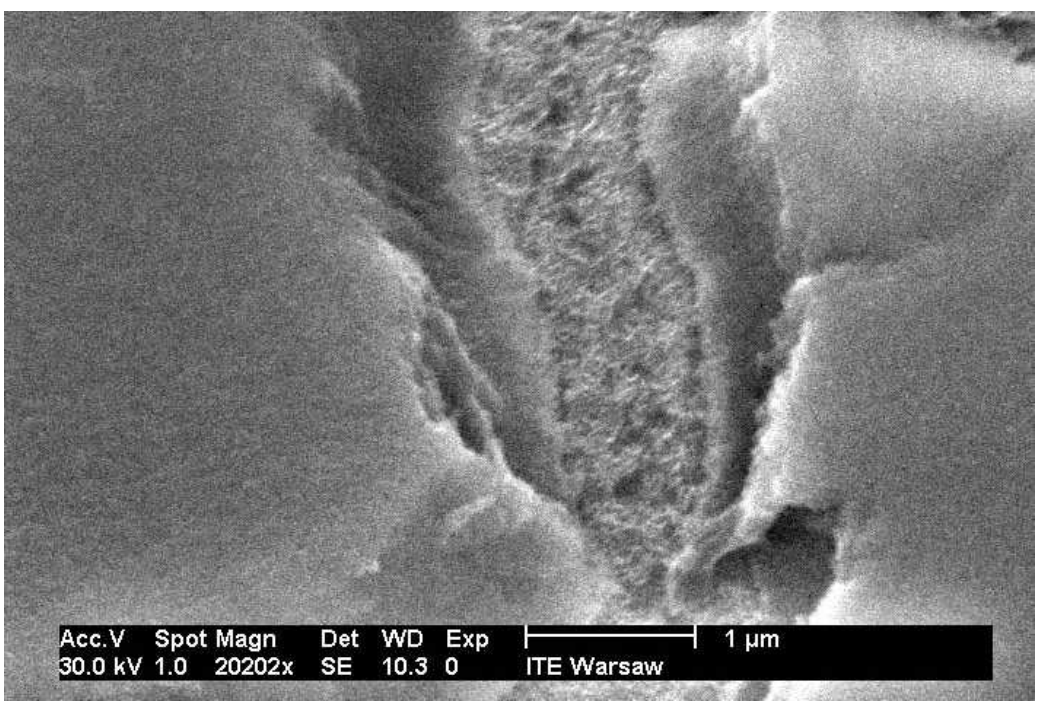

Fig. 1. SEM microphotograph of PSi layers. Near surface is a less porous auxiliary $\mathrm{PSi}^{*}$ layer, and the basic $\mathrm{PSi}^{* *}$ layer of greater porosity is seen in the depth. Aluminum contacts are not seen.

cross-section size of the silicon strings after etching is less than 10 nanometers at porosity of $75 \%$ [10].

$I-V$ characteristics of the structures are linear up to $2-3 \mathrm{~V}$ voltage (Fig. 2). Linearity shows that the contacts on both sides of the samples are ohmic. This is an essential difference of our structures from the ones known in references. The resistance of the samples varied within the limits of 50-200 Ohm. Experimental values of the resistance are lower than it should be according to the calculations. We explain these variations by the difference both in depth and density of breakings in PSi layer.

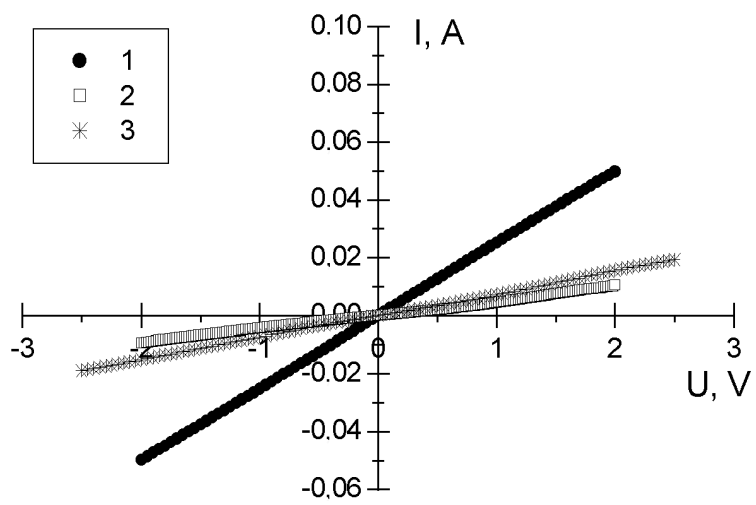

Fig. 2. Current-voltage characteristics of the structures. 
The investigation of the response to microwave radiation was performed on the samples in open-circuit mode without an external voltage, i.e. the arising electromotive forces (emf's) were studied. The action of the microwave radiation causes heating of free charge carriers. An emf can arise across a gradient of doping or accords geometrical non-uniformity of a structure as a result of non-uniform heating of free carriers $[11,12]$. In our case spatial geometrical heterogeneity exists on the interface between the $\mathrm{PSi}^{* *}$ layer and c-Si, or between two layers of different porosity $\left(\mathrm{PSi}^{*} / \mathrm{PSi}^{* *}\right.$ interface). Moreover, $p-p^{+}$homojunction exists in the interface of undoped and doped material. Hot carrier emf may be induced in all above-mentioned cases.

A response signal arises across our structures under the action of MW radiation. Let us first discuss the response at low values of microwave power: up to $1 \mathrm{~W}$ for samples 1 and 3, and up to $200 \mathrm{~mW}$ for sample 2 (Fig. 3). The signal pulse was of positive polarity, and basically repeating the shape of the stimulating microwave pulse. Usually, the recombination of carriers in silicon is lingering more than one $\mu \mathrm{s}$. Therefore, a short relaxation time of the response specifies that it is not related to the excitation of nonequilibrium charge carriers. The amplitude of the response depends on the microwave power as $U \sim P^{s}$ (Fig. 3). An exponent $s$ is within interval from 0.6 up to 1 . A short time of relaxation and linear dependence of the signal amplitude are characteristic of hot carrier emf in semiconductors $[11,12]$. Therefore, in our case the signal may be caused by the heating of free holes in our structures. Owing to heterogeneity of the structure, the heating of holes in the sample is non-uniform also. At low values of MW power, positive polarity of the signal implies the flow of heated holes from the porous side to the background of the structure. Considering the complex configuration of

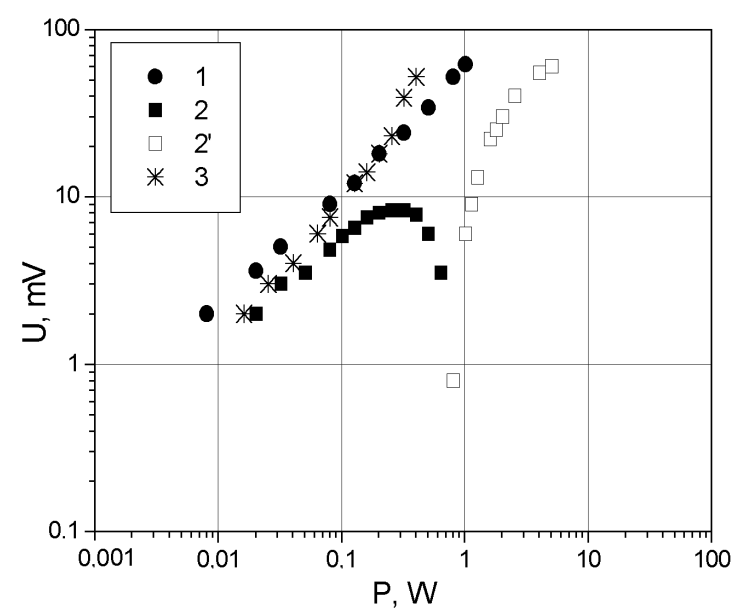

Fig. 3. Dependence of response amplitude across the structures on $10 \mathrm{GHz}$ microwave power. Full marks - positive signal, open squares $\left(2^{\prime}\right)$ - negative signal across the sample 2 . 
the structure, the signal in this case may be composed of hot carrier emf's of the same polarity induced across the junctions of interfaces such as $p^{+}-\mathrm{Si}^{*} / p-\mathrm{PSi}^{*}$ and $\mathrm{PSi}^{*} / \mathrm{PSi}^{* *}$.

The amplitude of the positive response on the sample 2 decreases as the MW power is further increased (see Fig. 3). At larger power values the signal changes its sign. The shape of the response pulse does not change, i.e. the negative signal is fast enough. It implies, therefore, that the response is still caused by the heating of free holes. Such hot carrier emf's of negative polarity may arise across the junction of $\mathrm{PSi}^{* *} / \mathrm{c}-\mathrm{Si}$ interface and across the homojunction $\mathrm{c}-\mathrm{Si} / p^{+}$-c-Si. The second emf should not considerably influence the signal formation due to two reasons. The first one, the large cross-sectional area of c-Si, compared to the porous part of silicon, is affected by much less concentrated electric field of the MW radiation. The second one, the conversion of the signal polarity is observed only on the sample of the highest resistance. It implies that the porosity of this sample is higher than that of the others, and as a result the geometrical non-uniformity of $\mathrm{PSi}^{* *} / \mathrm{c}-\mathrm{Si}$ interface should be higher in the sample 2 as well. Moreover, the heterojunction may be formed in this interface due to the possible formation of inverse layer. As a rule, the magnitude of hot carrier emf is proportional to the magnitude of inhomogeneity in a semiconductor [11]. Thus, the total signal under the action of MW radiation is composed of two sets of emf's of different polarity. For the sample 2, the amplitudes of the opposite signals are comparable at MW pulse power around $800 \mathrm{~mW}$. At higher power values, as the positive emf's get saturated, there prevails emf induced across the $\mathrm{PSi}^{* *} / \mathrm{c}-\mathrm{Si}$ interface. A slight sublinearity of the signal-power dependences for all samples at low power values can be explained by possible influences of negative emf induced across this interface.

\section{Conclusion}

Two-terminal structures are made of $p$-type silicon containing layers of the porous silicon between two metal contacts, supplied with additionally doped semiconductor regions near contact areas. It is established that current-voltage characteristics of the structures are linear. The response to the action of microwave radiation is investigated for the first time. The analysis of the basic characteristics of the response shows that it is caused by the heating of free holes and is composed of a set of emf's induced across the interfaces of the structure. Depending on the MW power, the polarity of the total signal depends on the emf component prevailing at the moment. We have shown for the first time that the structures containing porous silicon layers can be applied in a role of sensors of high frequency electromagnetic radiation.

\section{Acknowledgments}

The authors greatly acknowledge Department of Materials Semiconductor Structures Research, Institute of Electron Technology, Warsaw, for SEM microphotographs of structures. 


\section{References}

[1] L.T. Canham, Appl. Phys. Lett. 57, 1046 (1990).

[2] F. Namavar, H.P. Maruska, N.M. Kalhoran, Appl. Phys. Lett. 60, 2514 (1992).

[3] L.V. Beljakov, D.N. Gorjachev, O.M. Sreseli, I.D. Jaroshetsky, Semiconductors 27, 1371 (1993).

[4] A.V. Zherzdev, V.H. Kudojarova, A.V. Medvedev, G.K. Frost, Techn. Phys. Lett. 19, 87 (1993).

[5] N.J. Pulsford, G.L.J.A. Rikken, Y.A.R.R. Kessener, E.J. Lous, A.H.J. Venhuizen, J. Appl. Phys. 75, 636 (1994).

[6] K. Itotia, R.F. Drayton, in: Proc. IEEE MTT-S Int. Microwave Symp. Digest, Ed. R. Hamilton, Co-ed. N. Chiang, CD-ROM Ed. A. Taylor, IEEE Cat. No. 02CH37278, Vol. 2, Seattle ((WA) USA) 2002, p. 681.

[7] L.A. Balagurov, S.C. Bayliss, V.S. Kasatochkin, E.A. Petrova, B. Unal, D.G. Yarkin, J. Appl. Phys. 90, 4543 (2001).

[8] S.V. Slobodchikov, D.N. Gorjachev, H.M. Salihov, O.M. Sreseli, Semiconductors 33, 340 (1999).

[9] M.G. Berger, C. Dieker, M. Thönissen, L. Vescant, H. Lüth, H. Münder, W. Theiss, M. Wernke, P. Grosse, J. Phys. D, Appl. Phys. 27, 1333 (1994).

[10] L. Pavesi, Riv. Nuovo Cimento 20, 3 (1997).

[11] S. Ašmontas, in: Electrons in Semiconductors, Ed. J. Požela, Vol. 5, Vilnius 1984, p. 183 (in Russian).

[12] J.K. Pozhela, K.K. Repshas, Phys. Status Solidi B 27, 757 (1968). 